\title{
Percepção do pesquisador português em neurociências quanto ao compartilhamento de dados de pesquisa em repositórios
}

\section{Perception of the Portuguese researcher in neurosciences about the sharing of research data in repositories}

\section{Percepción del investigador portugués en neurociencias sobre el intercambio de datos de investigación en repositorios}

Viviane Santos de Oliveira Veiga | vivianesantosveiga@gmail.com
Fundação Oswaldo Cruz, Instituto de Comunicação e Informação Científica e Tecnológica em Saúde. Rio de Janeiro, Brasil.

Cícera Henrique da Silva | cicera.henrique@icict.fiocruz.br

Fundação Oswaldo Cruz, Instituto de Comunicação e Informação Científica e Tecnológica em Saúde. Rio de Janeiro, Brasil.

Maria Manuel Borges | $\underline{\text { mmb@fl.uc.pt }}$

Universidade de Coimbra. Coimbra, Portugal.

Paulo Roberto Borges de Souza Junior | paulo.borges@icict.fiocruz.br

Fundação Oswaldo Cruz, Instituto de Comunicação e Informação Científica e Tecnológica em Saúde. Rio de Janeiro, Brasil.

\section{Resumo}

Compartilhar dados de pesquisa é primordial para fornecer acesso a esses dados. Portanto, é essencial conhecer a percepção do pesquisador sobre o compartilhamento de seus dados de pesquisa, a fim de verificar as barreiras existentes e os estímulos que podem ser desenvolvidos para suportar a sua partilha. Para isso, uma pesquisa on-line foi realizada com 46 pesquisadores portugueses de neurociências. Verificou-se que a maioria dos entrevistados $(78,3 \%)$ nunca compartilhou dados de pesquisa em repositórios. Os motivos da retenção de dados foram: 1) perder oportunidades de publicação $(44,4 \%), 2)$ abusar ou interpretar dados $(41,7 \%), 3)$ ignorar repositórios para compartilhar, (6\%) e 4) falta de conhecimento sobre o possibilidade de abertura desses dados devido a informações confidenciais $(30,6 \%)$.

Palavras-chave: Dados de Pesquisa Abertos; Compartilhamento de Dados de Pesquisa; Ciência aberta. 


\section{Abstract}

Sharing research data is fundamental to providing access to this data. So, it's essential knowing the researcher's perception about sharing of their research data in order to verify the existing barriers and the stimuli that can be developed to support their sharing. For that, an online survey a research was conducted with 46 Portuguese neuroscience researchers. It was found that the majority of respondents (78.3\%) never shared research data in repositories. The reasons for data retention were: 1) to lose publication opportunities $(44.4 \%), 2)$ to misuse or interpret data $(41.7 \%), 3)$ to ignore repositories to share, $(6 \%)$ and 4 ) lack of knowledge about the possibility of these data being opened due to confidential information (30.6\%).

Keywords: Open research data; Research Data sharing; Open Science.

\section{Resumen}

Compartir datos de investigación es fundamental para proporcionar acceso a estos datos. Por lo tanto, es esencial conocer la percepción del investigador sobre el intercambio de sus datos de investigación con el fin de verificar las barreras existentes y los estímulos que se pueden desarrollar para apoyar su participación. Para ello, se realizó una encuesta en línea con una investigación de 46 investigadores portugueses en neurociencia. Se encontró que la mayoría de los encuestados $(78,3 \%)$ nunca compartieron datos de investigación en repositorios. Las razones para la retención de datos fueron: 1) perder oportunidades de publicación $(44,4 \%)$; 2) mal uso o interpretación de datos $(41,7 \%)$; 3) ignorar repositorios compartidos, (6\%) y 4) posibilidad de que estos datos se abran debido a información confidencial (30,6\%).

Palabras clave: Datos de investigación abiertos; Compartir datos de investigación; Ciencia abierta.

\section{Introdução}

Portugal é o país de língua portuguesa precursor na discussão do Acesso Aberto e na criação de repositórios. Esta trajetória de sucesso se iniciou com o Repositório da Universidade do Minho, o RepositoriUM, em 2003․ Este repositório institucional (RI) tem sido um "referencial ao desenvolvimento de outros repositórios, nomeadamente em Portugal e nos países de língua oficial portuguesa. Portugal conta com 48 RIs com coleção de artigo e destes $81 \%$ utiliza a opção do autoarquivamento ${ }^{2}$

Desde as primeiras iniciativas de compartilhamento de artigos científicos como as da biblioteca CiteSeeri, na década de 1980 e do Arxivii em 1991, passando pela proposta subversiva de Harnad, em 1994, publicada posteriormente pela Association of Research Libraries e as sequentes declarações da Comunidade Cientifica em prol do Acesso Aberto aos artigos científicos: a Budapest Open Access Initiative - BOAI $^{3}$, 2002, a Berlin ${ }^{4}$, em 2003 e a Bethesda 5 , também em 2003, tem multiplicado o número de repositórios, ampliando a visibilidade e o acesso aos resultados da pesquisa em forma de artigo. É notório o avanço na ampliação do acesso aos artigos científicos (que ainda tem um longo caminho a percorrer até alcançar os propósitos de abertura preconizados na $\mathrm{BOAI}^{3}$ ). Porém, outra tipologia integrante do processo de produção da ciência tem ocupado as discussões da comunidade acadêmica em prol do acesso aberto, os dados de pesquisa.

A enhanced Science ou e-Science ${ }^{6}$, considerada o $4^{\circ}$ Paradigma da Ciência refere-se não apenas à divulgação dos resultados, mas também a inclusão dos dados de pesquisa que lhes dão origem em repositórios. A e-Science também pode ser chamada de eResearch7. A "ciência aberta”, ou open Science, é um conceito guarda-chuva que abrange áreas como: dados abertos de pesquisa, publicação ampliada, artigo de dado (data paper), revisão por pares de dados, dados linkados, revisão por pares aberta, avaliação da ciência aberta (impacto e métricas abertas), recursos abertos (incluindo recursos educacionais abertos), software

\footnotetext{
i Atualmente CiteSeer ${ }^{x}$ é uma biblioteca digital de literatura científica e ferramenta de busca que se concentra principalmente na área da Computação e Ciência da Informação. http://citeseerx.ist.psu.edu/about/site.

ii Arxiv é um servidor de arquivamento e distribuição eletrônica de artigos científicos atualmente abrangendo as áreas de física, matemática, ciência da computação, ciências não lineares, biologia quantitativa e estatística.
} 
aberto, e outros. Estes termos exprimem uma nova lógica de disponibilização, de produção e de organização do conhecimento que assenta nas Tecnologias de Informação e Comunicação (TIC). Com o desenvolvimento das TICs a comunidade científica pôde criar, coletar e observar uma quantidade de dados, que anteriormente seria impossível. Além de trabalhar com esta grande quantidade de dados, as TICs também possibilitaram o seu compartilhamento, tanto no que respeita ao próprio artigo científico como no caso da publicação, onde o autor ao publicar seu artigo, pode incluir vários recursos (enhanced publication), não somente os dados ou bancos de dados de pesquisa, mas também vídeos, gráficos, wikis, blogs, etc. ${ }^{8-9}$

A figura 1 a seguir apresenta a evolução dos paradigmas da ciência desde a ciência empírica até os dias atuais com uma ciência baseada em dados, unificando teoria, experimento e simulação, a e-Science, que pode ser considerada uma "ciência em rede e baseada em dados", caracterizada pela ciência que se baseia em tecnologia computacional, com uso compartilhado de um volumoso e intenso conjunto de dados, para a realização de pesquisa colaborativa. Jonh Taylor foi o primeiro a utilizar o termo, em 2001, para se referir a uma "ciência crescentemente desenvolvida por meio de colaborações globalmente distribuídas viabilizadas pela Internet, usando grandes coleções de dados, recursos computacionais em escala tera e visualização de alto desempenho"10

Figura 1 - Paradigma da Ciência

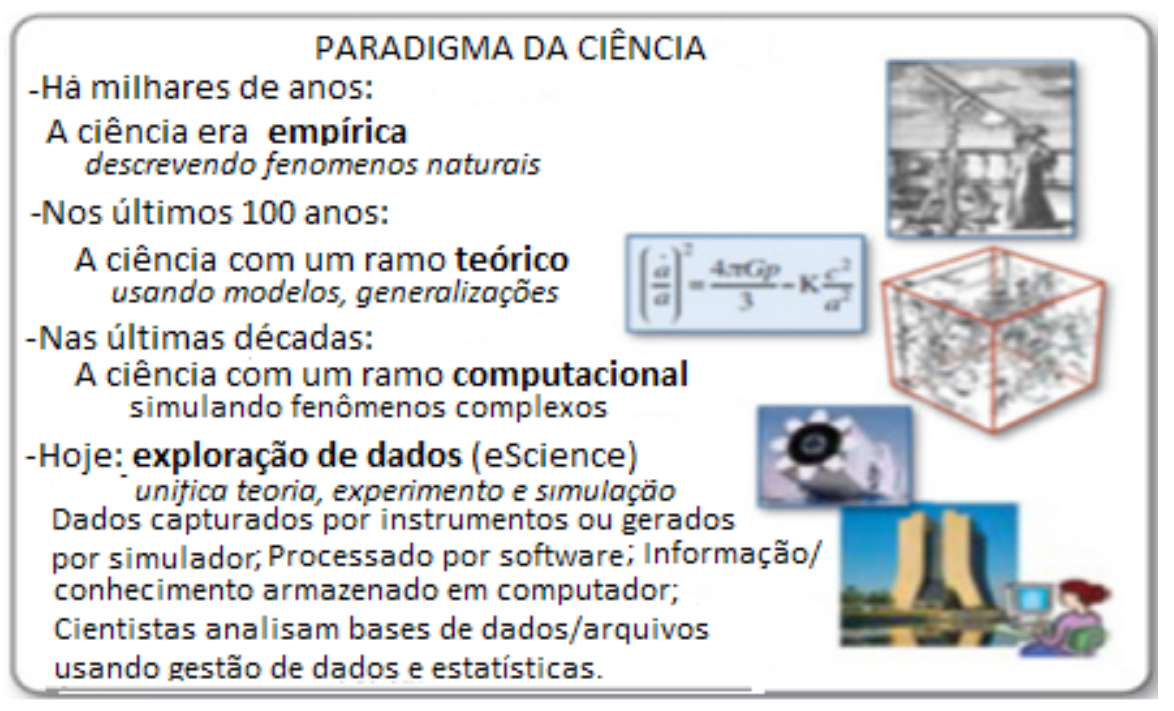

Fonte: Gray ${ }^{11}$ (tradução nossa)

Desde a Declaração de Berlim, em 2003, é recomendado o compartilhamento de dados de pesquisa em repositórios. Novas definições sobre o AA destacam os dados de pesquisa. A Diretoria Geral para a Pesquisa e Inovação da Comissão Européia salienta que "o acesso aberto à informação científica se refere a duas categorias principais: a) Publicações científicas revisadas por pares (...) b) Dados de pesquisa.

"Mudanças sistêmicas estão ocorrendo atualmente na forma como o sistema de ciência e pesquisa funcionam. Essas mudanças - referidas como "ciência aberta" - Implicam em uma forma de investigação mais aberta, colaborativa e em rede. $O$ acesso aberto é uma parte fundamental deste fenômeno. $O$ acesso aberto (AA) pode ser definido como a prática de fornecer acesso on-line à Informação gratuita para o leitor e reutilizável. No contexto da P \& D, o acesso aberto à 'informação científica' refere-se a duas categorias principais: a) Publicações científicas revisadas por pares (principalmente artigos publicados em Revistas acadêmicas) b) Dados de pesquisa (dados subjacentes às publicações e / ou outros dados conjuntos de dados curados mas inéditos ou dados brutos)”212. (tradução nossa) 
Neste contexto a Comisão Européia estabeleceu dois objetivos principais até 2020: todas as publicações científicas em revistas avaliadas por pares estarão acessíveis de forma aberta; e o compartilhamento de dados de pesquisa será o padrão para pesquisa científica.

Os dados de pesquisa para estarem abertos devem conter quatro requisitos fundamentais: acessibilidade, inteligibilidade, ser avaliável e utilizável ${ }^{13}$. A Royal Society, desde 2012, recomenda que as revistas cientificas implementem progressivamente o compartilhamento de dados de pesquisa como requisito para a publicação do artigos científicos. Várias revistas já têm implementado esta política, porém o seu cumprimento ainda precisa de monitoramento. Alguns periódicos, incluindo o grupo Public Library of Science (PloS), exigem, como condição para publicação, o compartilhamento de dados biomédicos detalhados para bases de dados de acesso aberto.

Em Portugal dos 10 periódicos AA da área médica registrados no Directory of Open Access Journals (DOAJ), 3 solicitam o depósito/publicação de dados. Estes depósitos podem ser de vários tipos de dados, sendo predominante nestes periódicos a exigência do registro dos ensaios clínicos, tanto no Brasil quanto em Portugal. Isto porque existem políticas mandatórias para estes dados. A maioria destes periódicos aponta a política da Organização Mundial da Saúde (OMS) e do Comitê Internacional de Editores de Revistas Médicas (ICMJE) como justificativa para a solicitação de depósito dos dados de pesquisa.

Uma pesquisa feita por Alsheikh-Ali et al ${ }^{14}$, com os 50 periódicos em biomedicina com maior fator de impacto identificou que 22 possuem uma política mandatória para o compartilhamento de dados como condição para publicação e outros 22 recomendam o depósito dos dados. Apesar disto, uma revisão feita nos primeiros 10 artigos publicados em cada periódico em 2009 (500 no total), revelou que apenas 9\% tinham efetivamente depositado os dados brutos completos

Em 2014, a União Europeia em suas diretrizes para o Programa Horizonte 2020 afirma que o acesso aberto às publicações e aos dados é um caminho que "envolve cidadãos e sociedade para melhorar a transparência do processo científico"15 (tradução nossa)

A National Science Foundation (NSF - Fundação Nacional de Ciência, Política de Compartilhamento de dados) tornou público por meio da NSF Data Sharing Policy ${ }^{16}$ sua recomendação para que "os pesquisadores compartilhem com outros pesquisadores, há um custo mínimo e dentro de um prazo razoável, os dados primários, amostras, coleções físicas e outros materiais de apoio reproduzidos ou reunidos no curso do trabalho sob concessão da NSF" (tradução nossa).

Desde 2003, o National Institute of Health (NIH) exigiu um plano de compartilhamento de dados para todos os grandes subsídios de financiamento ${ }^{17}$.

O tratato da Antartica prevê desde 1959 que "os resultados de observações científicas da Antarctica serão trocados e disponibilizados gratuitamente"18

Em 1996, com cientistas envolvidos no Projeto Genoma Humano, foram estabelecidos os Princípios das Bermudas (Bermuda Principles) onde a comunidade científica se reuniu para estabelecer regras para a liberação rápida e aberta de dados de pesquisa sobre sequências de DNA. Este esforço multinacional para seqüenciar o genoma humano gerou rapidamente uma grande quantidade de dados sobre a composição genética de seres humanos e outros organismos. Estes princípios exigiam que todos os dados da seqüência de DNA fossem liberados em bases de dados acessíveis ao público dentro de vinte e quatro horas após a sua geração e representam uma conquista significativa da transferência do que se pensava ser privado para bem público. Os princípios originais previam a liberação automática de conjuntos de seqüências maiores que 1 kb (preferencialmente dentro de 24 horas), a publicação imediata de sequências concluídas e tornar a seqüência inteira disponível abertamente, em domínio público, para pesquisa e desenvolvimento, maximizando os benefícios para a sociedade. Em 2005, a Organização de Cooperação e de Desenvolvimento Económico (OCDE) estabeleceu recomendações para que o acesso aberto aos dados de pesquisa com financiamentos público fosse fácil e rápido ${ }^{6}$. 
Também em 2005, é estabelecida a Global Earth Observation System of Systems (GEOSS) com o objetivo de criar princípios para o compartilhamento de dados de pesquisa sobre o meio ambiente. Dez anos depois estes princípios são confirmados e novas estratégias estabelecidas em seu Plano Estratégico 2016-2025, com o seguinte destaque para o compartilhamento de dados na GEOSS:

Dados, metadados e produtos serão compartilhados como dados abertos por padrão, tornandoos disponíveis como parte da Coleta de Dados GEOSS de Recursos Abertos para todos, sem encargos ou restrições de reutilização (...) e todos os dados, produtos e metadados compartilhados serão disponibilizados com um tempo mínimo ${ }^{19}$.

Segundo as diretrizes da Comissão Europeia, estabelecidas em 2016, o padrão, o comum, deve ser os dados de pesquisa financiada com recursos públicos, estarem abertos. Reconhecendo simultaneamente as necessidades de diferentes regimes de acesso devido aos direitos de propriedade intelectual, à proteção e confidencialidade dos dados pessoais e a competitividade econômica mundial e outros interesses legítimos, o dado de pesquisa deve estar "tão aberto quanto possível, e tão fechado quanto necessário" ${ }^{12}$. (tradução nossa)

O compartilhamento de dados de pesquisa é fundamental para prover o acesso a estes dados. No caso do artigo científico, a barreira na maioria das vezes é financeira, o que implica pagamento, da instituição ou do pesquisador, para obter acesso. No caso dos dados de pesquisa a barreira é de natureza diversa. Atualmente, na maioria dos casos, se o pesquisador não compartilhar seus dados nenhum pesquisador lhes poderá acessar, mesmo aqueles que disponham de recursos financeiros. A questão do compartilhamento dos dados de pesquisa perpassa também a questão do financiamento da ciência. Pesquisadores brasileiros envolvidos na investigação da gestão de dados abertos2o apontam: "o acesso e compartilhamento de dados de pesquisa contribui de forma significativa para que a ciência avance e maximize os investimentos aplicados em programas de pesquisa”.

O compartilhamento de dados traz vários benefícios para a comunidade científica, como estimular olhares de várias disciplinas e perspectivas de um determinado tema; possibilitar a identificação de erros de interpretação e fraudes; evitar desperdício de recursos financeiros; evitar retrabalho (nova coleta de dados). Em estudo publicado na PlosOne21 registra que o compartilhamento dos dados de pesquisa foi associado a um aumento de $69 \%$ nas citações, independentemente do fator de impacto do periódico, data de publicação e país de origem do autor, o que significa que o compartilhamento de dados traz benefícios também para o pesquisador. O compartilhamento de dados através do data paper (artigo de dado) possibilita o reconhecimento do criador/coletor dos dados de forma citável apoiando os processos de avaliação da ciência e do sistema de recompensa acadêmica e de pesquisa. O principal objetivo de um artigo de dado é descrever os dados, e não o resultado da pesquisa, da análise realizada com base nos dados ${ }^{22}$.

Porém, muitas são as barreiras na visão do pesquisador para o compartilhamento de dados. Este pressuposto foi instigador para a realização de investigação no doutorado recém finalizado, quando foram mapeadas as barreiras na percepção dos pesquisadores na área de neurociências em Portugal.

Sendo assim, o objetivo deste trabalho é apresentar algumas justificativas dos neurocientistas em Portugal para a retenção de dados de pesquisa.

\section{Metodologia}

A fim de identificar os principais motivos para a retenção dos dados de pesquisa foi construído um questionário, utilizando a planilha do Google Forms. Este questionário foi enviado para 333 pesquisadores de neurociências do Centro de Neurociências e Biologia Celular (CNC), da Universidade de Coimbra e do 
Instituto de investigação e inovação em saúde (I3S/IBMC). Foram retornados 46 questionários respondidos, no período de janeiro a março de 2016.

Para a análise dos dados quantitativos do questionário utilizaram-se duas ferramentas. Na primeira etapa os dados foram extraídos do GoogleForms para uma planilha no programa Microsoft Excel e importados para o programa Statistical Package for the Social Sciences (SPSS), versão 22, onde foram calculadas médias e proporções e construídas as tabelas de frequências e cruzamentos. Na segunda etapa as tabelas foram exportadas para planilhas no programa Microsoft Excel onde foram desenvolvidos os gráficos.

\section{Resultados}

Verificou-se que a maioria dos respondentes $(78,3 \%$ dos pesquisadores) nunca compartilhou dados de pesquisa em repositórios. (Gráfico 1)

Gráfico 1 - Compartilhamento de dados de pesquisa em repositórios $(N=46)$

Já partilhaste, ou alguém em seu nome, dados de pesquisa em um repositório institucional ou temático?

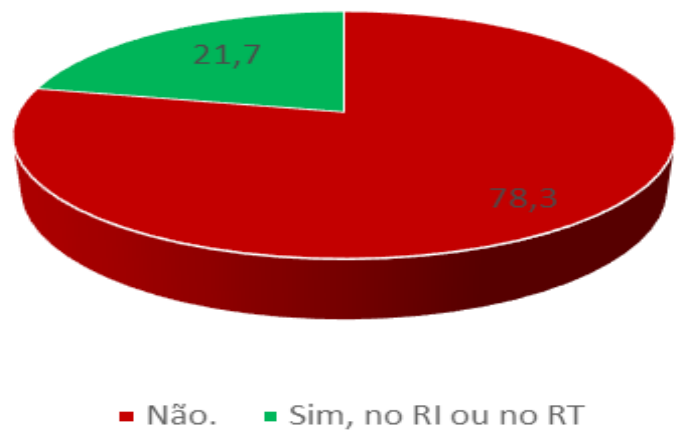

Fonte: Dados da pesquisa

Percebe-se neste gráfico acima a importância do estudo sobre compartilhamento e retenção de dados de pesquisa. Apenas 11 neurocientistas, dentre os 46 respondentes já compartilharam seus dados em repositórios digitais. É imperativa a investigação sobre as barreiras enfrentadas pelos pesquisadores para o compartilhamento dos dados, Desta forma será possível criar mecanismos que minimizem as dificuldades e facilitem o amplo acesso aos dados de pesquisa. No gráfico 2, a seguir, pode-se visualizar que perder oportunidades de publicações $(44,4 \%)$ e a má utilização ou interpretação dos dados $(41,7 \%)$ foram as principais justificativas para o não compartilhamento de dados de pesquisa em repositórios entre os respondentes. O desconhecimento de repositórios para fazer o compartilhamento e sobre a possibilidade destes dados estarem abertos devido a informações sigilosas, também foram alegados como justificativa por 30,6\% dos pesquisadores.

As preocupações com perdas de oportunidade de publicação e com direitos exclusivos sobre seus dados são fatores que podem influenciar no comportamento do pesquisador em compartilhar dados em Acesso Aberto. Nesta pesquisa 44,4\% dos respondentes alegou este motivo para a retenção dos dados. Este resultado corrobora os achados de Sedberry ${ }^{23}$ que em sua pesquisa verificou que pesquisadores não compartilham seus dados por medo de outro pesquisador utilizar seus dados e publicar antes deles. Na literatura da área foram identificados estudos ${ }^{24-2526}$, em que pesquisadores afirmaram que não compartilham seus dados para que possam ter mais oportunidades de publicação. Eles preferem manter seus dados exclusivos para serem reutilizados por eles mesmos ou por seus alunos em novas publicaçoes. Tenopir ${ }^{27}$ verificou que os pesquisadores 
também consideram o compartilhamento de dados como uma perda de oportunidade de comercialização dos dados. A autora relatou também que em disciplinas onde existe maior oportunidade em comercializar dados ou achados de pesquisa e/ou requerer patentes existe maior tendência para a retenção dos dados.

Nesta pesquisa em Portugal, verificou-se que muitos pesquisadores reutilizam seus próprios dados para desenvolvimento de diversos artigos e o compartilhamento destes dados ainda não totalmente explorados aparece como um custo que interfere no compartilhamento. Outra questão se relaciona à vantagem competitiva.

\section{Gráfico 2 - Justificativas para não compartilhar dados de pesquisa $(N=35)$}

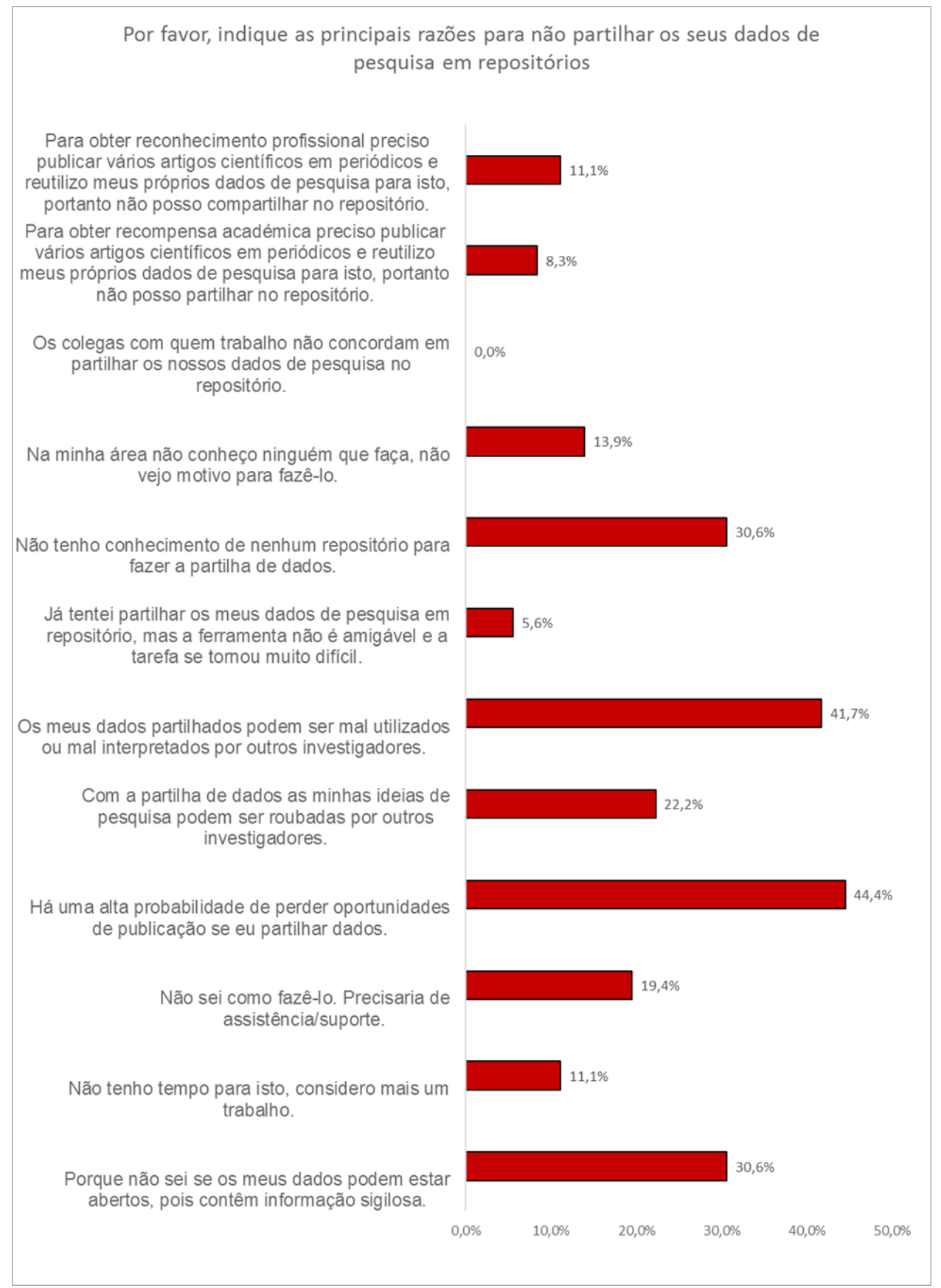

Fonte: Dados da pesquisa

*Proporção baseada em questão de múltiplas respostas.

Os pesquisadores se preocupam em compartilhar seus dados e isto atribuir uma vantagem a outro pesquisador em publicar sobre os seus dados antes do próprio. Os pesquisadores afirmam que para a coleta e 
organização destes dados é empregado muito trabalho e tempo e eles se ressentem de entregar seus dados para outro pesquisador que usufruirá destes dados sem nenhum esforço e reconhecimento dos coletores.

Estes temores seriam minimizados se estes pesquisadores conhecessem sobre os serviços disponíveis para a identificação do coletor/produtor dos dados de pesquisa. Existe a possibilidade dos dados receberem um Identificador persistente, seja pelo repositório, seja pelos serviços de atribuição de Digital Object Identifier (DOI) para os dados de pesquisa, seja pelos data papers. São mecanismos de citação de dados de pesquisa para reconhecimento dos seus produtores.

A preocupação com a má utilização ou interpretação dos dados de pesquisa por outros pesquisadores é uma barreira que influencia o pesquisador no comportamento de compartilhamento de dados de pesquisa. Nesta pesquisa $41,7 \%$ dos respondestes justificaram a retenção pela preocupação com má utilização ou interpretação dos seus dados. Estes achados corroboram os achados de outros autores, assim eexplicitados:. Segundo Stanley preocupação com o mau uso que seus dados podem sofrer na mão de pesquisadores inexperiente ${ }^{28}$,. com o uso inadequado dos dados compartilhados, com uma interpretação incorreta dos $\operatorname{mesmos}^{29}{ }^{23}$. O medo de ser plagiado ou ter seus dados roubados também afasta o pesquisador do compartilhamento pela via verde ${ }^{31}$. Também evidenciaram que os pesquisadores se preocupam em liberar os resultados antes de um artigo ser publicado. Liberar os resultados antes da publicação formal foi equiparado a dar uma vantagem competitiva sobre as descobertas.

Para minimizar esta barreira que tem influenciado o comportamento de compartilhamento do pesquisador faz-se necessário que sejam criados repositórios confiáveis com políticas de uso claras que resguardem o coletor/produtor dos dados, para que a confiança adquirida pelos pesquisadores nos repositórios para o depósito de artigos alcance também a coleção de dados de pesquisa.

O desconhecimento sobre a existência de repositórios é algo que carece de atenção dos profissionais de informação. Apesar de todo esforço na criação de dispositivos, como o repositório, para o compartilhamento de artigos e dados, há registros na literatura de que nem sempre existe uma boa divulgação dos mesmos. O não compartilhamento da produção no repositório institucional porque não sabiam da existência dele é motivo alegado em pelo menos dois estudos, sendo um português ${ }^{32} \mathrm{e}$ um espanhol ${ }^{33}$ No caso desta pesquisa verificou-se que 30,6\% dos pesquisadores não compartilhavam seus dados de pesquisa porque não conhecem repositórios para fazê-lo.

Os pesquisadores também justificaram nesta pesquisa a retenção de dados de pesquisa devido à preocupação com informação sigilosa. Em algumas áreas do conhecimento o compartilhamento de dados é limitado pois lida-se com dados sigilosos ${ }^{16}{ }^{34}$. Para estes autores, em áreas como a biomedicina e a assistência à saúde, o uso de dados de pacientes limita as possibilidades de compartilhamento. Para compartilhar dados de pesquisa envolvendo seres humanos é necessário todo um trabalho com os metadados de forma a proteger a identidade dos sujeitos das pesquisas. Existem várias formas de se trabalhar e organizar os dados de forma a manter em sigilo o que precisa realmente estar em acesso restrito, pois os dados de pesquisa devem estar "tão aberto quanto possível, e tão fechado quanto necessário" 35 . Uma outra questão que preocupa cientistas é o termo de consentimento assinado pelos sujeitos da pesquisa. A maioria destes acordos não estabelecem autorização para reutilização dos dados coletados na pesquisa para outras pesquisas ${ }^{23}$.

\section{Considerações finais}

Vivencia-se uma época em que se procura estimular a produção científica através de uma abordagem que implica uma maior partilha e construção conjunta de conhecimento. Para que efetivamente o investimento em conhecimento seja reutilizado e reinterpretado é necessário que todas as partes interessadas percebam a importância do compartilhamento dos resultados da pesquisa e dos dados que lhe dão origem e as 
vantagens que daí resultam para todos. E para que isso aconteça é também necessário procurar esclarecer todas as questões que se colocam num caminho que se trilha pela primeira vez.

Os pesquisadores que nunca compartilharam dados de pesquisa percebem o compartilhamento como um ônus, que pode lhe custar perda de oportunidades de publicação, ou má utilização dos seus dados. Percebe-se também que uma parcela significativa dos pesquisadores justifica a retenção dos dados por falta de conhecimento de um lugar para compartilhar. Instrumentos que garantam a prioridade de publicação dos dados de pesquisa compartilhados devem ser desenvolvidos para resguardar o autor dos dados. As ferramentas de citação de dados, bem como os artigos de dados, devem ser amplamente divulgadas. A criação de repositórios de dados de pesquisa utilizando protocolos e padrões internacionais de interoperabilidade para armazenar e disponibilizar os dados de pesquisa com descrições que garantam o reuso deve ser uma prioridade para o campo da informação.

\section{Referências}

1. Rosa FG, Gomes MJ. Coordenadores de comunidades de repositórios institucionais: o caso do repositoriUm. 2010 2. Sem. [citado em 2 ago. 2017] Enc. Bibli: R. Eletr. Bibliotecon. Ci. Inf.; ed.esp: Disponível em: http://www.periodicos.ufsc.br/index.php/eb/article/view/16591/15765.

2. Veiga, VSOV; Macena, LGG, Silva, CH, Borges, MM. Panorama do autoarquivamento nos repositórios institucionais portugueses. Cadernos BAD. 2016 [citado em 21 mar. 2017]; 2:96-105. Disponível em: https://www.bad.pt/publicacoes/index.php/cadernos/article/viewFile/1586/pdf.

3. Budapest Open Acces Initiative. [Internet]. 2002. Disponível em: <http://www. budapestopenaccessinitiative.org/read>. Acesso em: 08 mar. 2016

4. Berlin Declaration. Berlin Declaration on open access to knowledge in the sciences and humanities. 2003 [citado em 2 ago. 2017]. Disponível em: https://openaccess.mpg.de/67693/BerlinDeclaration pt.pdf.

5. Bethesda statement on open access publishing, 20 June 2003, Disponível em: http://legacy.earlham. edu/ peters/fos/bethesda.htm

6. Hey T,Tansley S, Tolle K. (Org.) The fourth paradigm: data-intensive scientific discovery. Redmond, Washington: Microsoft Research, 2009.

7. Anderson T, Kanuka H. E-research: methods, strategies, and issues. Boston: Allyn \& Bacon, 2003.

8. Verhaar P. Report on object models and functionalities. In: Place, Thomas et al. (Ed.). DRIVER II. 2008.

9. Vernooy-Gerritsen M. Emerging standards for enhanced publications and repository technology: survey on technology. Amsterdam: University Press, 2009. (Driver and Surf studies).

10. Fry J.; Schroeder R.; Den Besten M. Open science in e-science: contingency or policy? Journal of Documentation [Internet]. 2009 [citado em 2 ago. 2017]; 65(1):6-32. Disponível em: http://www. emeraldinsight.com/doi/pdfplus/10.1108/00220410910926103.

11. Gray J. Jim Gray on eScience: a transformed scientific method. In: Hey T.,Tansley S., Tolle K. (Org.) The fourth paradigm: data-intensive scientific discovery. Redmond, Washington: Microsoft Research, 2009.

12. European Commission. Directorate-general for research \& innovation. H2020 programme: guidelines on FAIR data management in Horizon 2020. Version 3.0. 2016 [citado em 2 ago. 2017]. Disponível em: http://ec.europa.eu/research/participants/data/ref/h2020/grants manual/hi/oa pilot/h2020-hi-oa-datamgt en.pdf.

13. The Royal Society. Science as an open enterprise: open data for open science. The Royal Society Science Policy Centre Report 02/12. [citado em 2 ago. 2017].The Royal Society, jun. 2012. Disponível em: http:// royalsociety.org/uploadedFiles/Royal Society Content/policy/projects/sape/2012-06-20-SAOE.pdf.

14. Alsheikh-Ali AA, Qureshi W, Al-Mallah MH, Ioannidis JPA. Public availability of published research data in high-impact journals. PLoS ONE [Internet]. 2011 [citado em 2 ago. 2017]; 6(9):e24357. Disponível em: http://journals.plos.org/plosone/article?id=10.1371/journal.pone.0024357.

15. European Commission. Directorate-General for Research \& Innovation. H2020 Programme: Guidelines on open access to scientific publications and research data in Horizon 2020. Version 3.0. 2016 [ citado em 2 ago. 2017]. Disponível em: http://ai.tecnico.ulisboa.pt/files/sites/52/guidelines-scientificpublicationsresearch-data-in-h2020.pdf 
16. National Science Foundation. Dissemination and sharing of research results. Disponível em: https:// www.nsf.gov/bfa/dias/policy/dmp.jsp Acesso em: 18 fev. 2017

17. National Institutes of Health. NIH Data sharing policy and implementation guidance. [citado em 2 ago. 2017]. Disponível em: https://grants.nih.gov/grants/policy/data_sharing/data sharing guidance.htm.

18. The Antarctic Treaty. [citado em 2 ago. 2017]. Disponível em: http://www.ats.aq/e/ats.htm.

19. Group on Earth Observations. GEO strategic plan 2016-2025: implementing GEOSS. 2016 [citado em 2 ago. 2017]. Disponível em: https://www.earthobservations.org/documents/GEO Strategic Plan 20162025 Implementing GEOSS.pdf.

20. Sayão LF; Sales LF. Guia de Gestão de Dados de Pesquisa para Bibliotecários e Pesquisadores. Rio de Janeiro: CNEN/IEN, 2015 [citado em 2 ago. 2017]. Disponível em: http://www.cnen.gov.br/images/CIN/ PDFs/GUIA DE DADOS DE PESQUISA.pdf

21. Piwowar HA, Day RS; Fridsma DB. Sharing detailed research data is associated with increased citation rate. PLoS ONE [Internet]. 2007 [citado em 20 mar. 2017]; 2(3):e308. Disponível em: http://journals. plos.org/plosone/article/file?id=10.1371/journal.pone.0000308\&type=printable.

22. Chavan V, Penev L. The data paper: a mechanism to incentivize data publishing in biodiversity science. BMC Bioinformatics [Internet]. 2011, [citado em 2 ago. 2017]; 12( SUPP. 15). Disponível em: https:// bmcbioinformatics.biomedcentral.com/articles/10.1186/1471-2105-12-S15-S2.

23. Sedberry GR, Fautin DG, Feldman M, Fornwall MD, Goldstein P, Guralnick RP. 2011. OBIS-USA: A datasharing legacy of the census of marine life. Oceanography [Internet]. 2011, [citado em 2 ago. 2017]; 24(2):166-173. Disponível em: http://tos.org/oceanography/assets/docs/24-2 sedberry.pdf.

24. Reidpath DD, Allotey PA. Data sharing in medical research: an empirical investigation. Bioethics [Internet] 2001, [citado em 2 ago. 2017];15:125-34. Disponível em: doi:10.1111/1467-8519.00220 [PubMed].

25. Campbell EG., Clarridge BR, Gokhale NN., Birenbaum L, Hilgartner S, Holtzman NA et al. Data withholding in academic genetics: evidence from a national survey. Jama-Journal of the American Medical Association [Internet] 2002 Jan, [citado em 2 ago. 2017]; 287(4):473-480.

26. Savage $\mathrm{CJ}$, Vickers AJ. Empirical study of datasharing by authors publishing in PLoS journals. PLoS ONE [Internet] 2009, [citado em 2 ago. 2017]; 4(9), e7078. Disponível em: http://journals.plos.org/plosone/ article?id=10.1371/journal.pone.0007078

27. Tenopir C, Allard S, Douglass $K$, Aydinoglu AU, Wu L, Read A et al. Data sharing by scientists: practices and perceptions. PLOS ONE [Internet] 2011, [citado em 2 ago. 2017]; 6(6): e21101. Dispoível em: https://doi.org/10.1371/journal.pone.0021101

28. Stanley B, Stanley M. Data sharing: the primary researcher's perspective. Law and Human Behavior. 1988; 12(2):173-180.

29. Borgman CL. Scholarship in the digital age: information, infrastructure, and the internet. Cambridge: MIT Press, 2007.

30. Cragin MH, Palmer CL, Carlson JR, Witt M. Data sharing, small science and institutional repositories. Phil. Trans. R. Soc. [Internet] 2010, [citado em 2 ago. 2017]; 368:4023-4038. Disponível em: http://rsta. royalsocietypublishing.org/content/368/1926/4023.

31. Davis PM; Connolly MJL. Institutional repositories: evaluating the reasons for non-use of Cornell University's installation of DSpace. D-Lib Magazine [Internet] 2007;13(3-4). Disponível em: http://www. dlib.org/dlib/march07/davis/03davis.html.

32. Rodrigues MEP, Rodrigues AM. Os autores e o repositório científico: estudo de caso. Rev Eletron de Comum Inf Saúde [Internet]. 2014 Set [citado em 2 ago. 2016]; 8(2): 107-121. DOI: 10.3395/reciis. v8.i2.912.pt

33. Borrego A. Institutional repositories versus Research Gate: the depositing habits of spanish researchers. Learned Publishing, [Internet] 2017, [citado em 2 ago. 2017]. Disponível em: doi:10.1002/leap.1099.

34. Lane J, Schur C. Balancing access to health data and privacy: a review of the issues and approaches for the future. Health Services Research [Internet]. 2010 [citado em 2 ago. 2017]; 45(5):1456-1467.

35. European Commission. Directorate-General for Research \& Innovation. Background note on open access to scientific publications and open research data [Internet], 2016 [citado em 2 ago. 2017]. Disponível em: https://ec.europa.eu/research/openscience/pdf/openaccess/background note open acces s.pdf. 